\section{Cost considerations in determining the affordability of adjuvant trastuzumab in breast cancer}

To the Editor: Roche (Pty) Ltd would like to comment on the article 'Cost considerations in determining the affordability of adjuvant trastuzumab in breast cancer' by Prof. Abratt. ${ }^{[1]}$

Trastuzumab is indicated in South Africa (SA) for the treatment of human epidermal growth factor receptor 2 (HER2)-positive metastatic breast and gastric cancer, as well as HER2-positive early breast cancer ${ }^{[2]}$ Large multinational studies evaluating the product in over 18000 patients with early HER2-positive breast cancer have shown marked long-term clinical benefit in this population. This treatment represents the largest degree of therapeutic benefit in early breast cancer reported since the introduction of tamoxifen for hormone-receptor-positive disease approximately 25 years ago. ${ }^{[3]}$ One year of treatment with trastuzumab is associated with a significant and sustained reduction in the risk of disease recurrence or death, irrespective of a patient's nodal status, hormone-receptor positivity, previous (neoadjuvant) chemotherapy regimen and age. ${ }^{[3.5]} \mathrm{A}$ recent update covering over 10 years' (median 11 years) follow-up of the pivotal HERA trial of patients treated with trastuzumab for 1 year showed an approximately $25 \%$ reduction in the incidence of disease-free and overall survival (OS) events when compared with the chemotherapy-only arm. Trials with a shorter duration of trastuzumab treatment of $<1$ year have to date failed to show noninferiority to 1 year of trastuzumab.

Updated results from these and other ongoing trials are not promising but have yet to conclusively determine whether shorterduration trastuzumab is an efficacious option. ${ }^{[6,7]}$ International guidelines confirm that 1 year of trastuzumab remains the standard of care as part of an adjuvant therapy regimen for patients with HER2positive early breast cancer. ${ }^{[8-10]}$

We note the following specific issues in relation to the commentary: 1. Parameters used in the number needed to treat (NNT) calculation:

The author notes that more sophisticated or accurate methods are available.

Survival at 10 years of patients who are treated with adjuvant trastuzumab is approximately $81 \%{ }^{[5]}$

A more accurate estimate of baseline survival at 10 years is derived if the actual 10-year survival of those who are eligible for adjuvant trastuzumab (the control arm from HERA) are used. Using this value of $75 \%$ the NNT is calculated as 17 , about half the value presented in the commentary.

The author states 'The HR [hazard ratio] is the ratio of the relative survival of two patient groups, with and without the test therapy. This ratio will not vary much over time. ${ }^{\text {?[1] }}$ This result has been shown to be broadly truthful for adjuvant trastuzumab treatment for HER2-positive breast cancer. It is, however, more frequently the case that the HR increases over time, showing reduced benefits from specific treatments.

2. Cost as a single basis for decision-making

By using the NNT for OS and simply multiplying this by the drug cost, there is an implication that OS at 10 years is the only benefit from this treatment. There are other very important financial and clinical benefits for patients treated with trastuzumab, most notably prevention of local, regional and distant recurrences, as well as additional years of disease-free life gained. Those treated are significantly more likely to be disease free for longer and, given that the baseline disease-free survival (DFS) rates are of course less than the baseline survival rate (BLSR), this leads to a profound reduction in the NNT.
The high costs, quality-of-life impacts, and debilitating consequences of recurrent disease suggest that prevention of recurrence is of considerable importance, in addition to the benefits of increased survival. ${ }^{[11]}$

Using DFS as the endpoint of focus is most appropriate in settings where it is expected that recurrence of disease presents the major component of mortality in the treated population. This would be the case for most solid tumours, for which secondary therapies at the time of recurrence may prolong survival but ultimately are unlikely to result in a full remission. DFS is most relevant when there is an extended interval between recurrence and death, thus requiring a longer follow-up for a primary trial endpoint of OS. ${ }^{[12]}$ This contrasts with complete response, partial response, or stable disease, which are considered meaningful in the neoadjuvant setting. ${ }^{[13,14]}$

In light of these comments, it is Roche's position that in order to informatively and appropriately make scientifically based local or policy-level best-treatment recommendations, the following as a minimum are required:

- a careful and thorough systematic literature search

- accumulation of true local cost information

- a robust review of the evidence with regard to clinical benefits and potential harms of the treatment options

- a robust cost-effectiveness analysis incorporating quality-of-life measures and all pertinent costs over a relevant post-treatment interval

- an evaluation of the personal and financial impacts at a patient level (ability to work, maintain family roles and responsibilities, self-care, and productivity) and country level (economic impact, burden of disease on healthcare system).

The far-reaching social and financial consequences of recommendations relating to novel treatments mean that these decisions need to be made in a robust, transparent and objective manner.

The approximation of costs as published in this article ${ }^{[1]}$ is inaccurate and potentially misleading for an intervention that provides DFS benefit for HER2-positive women, particularly if all the benefits and harms of the treatment are to be accounted for.

\section{Maksym Sereda}

Head: Medical Affairs Management Centre South Africa

Roche Products (Pty) Ltd Medical Department, Sandton, South Africa maksym.sereda@roche.com

\footnotetext{
. Abratt RP. Cost considerations in determining the affordability of adjuvant trastuzumab in breas cancer. S Afr Med J 2016;106(10):981-982. http://dx.doi.org/10.7196/SAMJ.2016.v106i10.11141

. Roche Products (Pty) Ltd. Herceptin Package Insert, South Africa. June 2016.

3. Untch M, Gelber RD, Jackisch C, et al. Estimating the magnitude of trastuzumab effects within patien

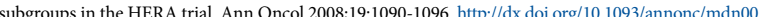

Piccat-G her MJ Procter M, Ley

Piccart-Gebhart MJ, Procter M, Leyland-Jones B, et al., Herceptin Adjuvant (HERA) Trial Stud Team. Trastuzumab after adjuvant chemotherapy in HER2-positive breast cancer. N Engl J Med
2005;353(16):1659-1672. http://dx.doi.org/10.1056/nejmoa052306 2005;353(16):1659-1672. http://dx.doi.org/10.1056/nejmoa052306

5. Jackisch C, Piccart MJ, Gelber RD, et al. HERA trial: 10 years follow up of trastuzumab after adjuvant chemotherapy in HER2 positive early breast cancer - Final analysis. Oral presentation at: San Antonio Breast Cancer Symposium 2015; 10 December 2015; San Antonio, Texas.

6. Pivot X, Romieu G, Debled M, et al. 6 months versus 12 months of adjuvant trastuzumab for patients with HER2-positive early breast cancer (PHARE): A randomised phase 3 trial. Lancet Oncol 2013; 14:741-748. http://dx.doi.org/10.1016/s1470-2045(13)70225-0

Sher or vinorelbine with or without trastuzumab for breast cancer. N Engl I Med 2006:354:809-820. http:/
(a) or vinorelbine with or without tras
dx.doi.org/ $10.1056 /$ nejmoa 053028

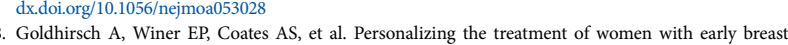
cancer: Highlights of the St Gallen International Expert Consensus on the Primary Therapy of Early Breast Cancer 2013. Ann Oncol 2013;24:2206-2223. http://dx.doi.org/10.1093/annonc/mdr304

9. Curigliano G, Cardinale D, Suter T, et al. Cardiovascular toxicity induced by chemotherapy, targeted agents and radiotherapy: ESMO Clinical Practice Guidelines. Ann Oncol 2012;23(Suppl 7):viil55-66. http://dx.doi.org/10.1093/annonc/mds293

10. Gradishar WI, Anderson BO, Balassanian R, et al. Invasive Breast Cancer Version 1.2016, NCCN Clinical Practice Guidelines in Oncology. J Natl Compr Canc Netw 2016;14(3):324-354
} 
11. Sargent DJ, Wieand HS, Haller DG, et al. Disease-free survival versus overall survival as a primary end point for adjuvant colon cancer studies: Individual patient data from 20898 patients on 18 randomized trials. J Clin Oncol 2005;23:8664-8670. http://dx.doi.org/10.1200/ico.2005.01.6071

12. Gill S, Sarge D. End points for adjuvant therapy trials: Has the time come to accept disease-free survival as a surrogate end point for overall survival? Oncologist 2006;11:624-629. http://dx.doi.org/10.1634/ theoncologist.11-6-624

13. Wardley AM, Cameron DA, Bell R, et al. Modelling predicts a long-term benefit from trastuzumab (Herceptin ${ }^{\circ}$ ) use in HER2-positive breast cancer. Poster Presentation at 31st ESMO Congress, 29 September - 3 October 2006; Istanbul, Turkey.

14. Murthy RK, Varma A, Mishra P, et al. Effect of adjuvant/neoadjuvant trastuzumab on clinical outcomes in patients with HER2-positive metastatic breast cancer. Cancer 2014;120(13):1932-1938. http://dx.doi. org/10.1002/cncr.28689

Prof. Raymond Abratt responds: The vast majority of patients with human epidermal growth factor receptor (HER)-positive breast cancer in South Africa (SA) do not have access to and do not receive adjuvant trastuzumab, be they cared for in the public sector or the less well-resourced medical scheme options in the private sector. The letter commenting on the article on cost considerations in determining the affordability of adjuvant trastuzumab in $\mathrm{SA}^{[1]}$ is founded on guidelines for patient care in affluent healthcare systems only. In such systems, trastuzumab is affordable upfront for patients. The letter ignores patients in less well-off healthcare systems. To rationally plan for quality healthcare for all patients in SA, we need to address drug access and its related budget impact, affordability and value.

The original article fully recognises the clinical activity of trastuzumab. It notes that baseline survival rate (BLSR) of patients (the survival rate without adjuvant trastuzumab) depends on their prognostic group. The expected survival rate for the different prognostic groups with adjuvant trastuzumab can be calculated with the hazard ratio (HR). The number needed to treat (NNT) to benefit one patient can then be determined. The drug costs, which are the major factor determining affordability, to benefit one patient are described for different prognostic groups.

This provides data to initiate a rational discussion of patient benefit from adjuvant trastuzumab, in healthcare systems with finite resources. The ethical principle of doing the best for all patients with the available resources is described.

There are scientific inaccuracies in the letter which need to be answered, even though it would be preferable to focus on improving patient care.

1. The letter comments on the parameters used in NNT calculation in the original article:

a. The author of the letter does not appreciate that calculations in the original article were done using the more sophisticated method and is referenced. The method of approximation was described in addition as it is helpful to clinicians.

b. The author of the letter quotes a single 10-year survival rate of adjuvant trastuzumab and ignores prognostic groupings which result in different survival rates and have different cost implications in the NNT to benefit one patient.

c. The author of the letter uses the 10-year survival rate from a single study rather than using all the scientific information from studies, which informs the HR. Moreover, the author states that with a BLSR of 75\%, the NNT to benefit one patient is 17 and that the value in the original article is double that. This is incorrect. In the original article the table indicates that for a BLSR of $80 \%$, the NNT $=17.5$ and for a BLSR of $70 \%$, the NNT $=12.2$, which is similar to or lower than that in the letter.

d. The comment in the letter about HR is tangential and does not impact on the calculations in the original article. The assumption in the Cox model is that the HR is constant over time. It is indeed possible for it not to be so. If after a while, there is a substantial difference in the proportion of patients who are completely cured in one arm of a trial, they will no longer be experiencing the hazard (death) at the same rate as they were before. The control group may be dying (experiencing the hazard) at the same rate as before and so the HR will be different from what it has been earlier. However, the differences in cure rates between the two arms is relatively small, particularly in the clinical range described.

2. The letter notes that trials with a duration of trastuzumab treatment of $<1$ year have, to date, failed to show non-inferiority to 1 year of trastuzumab. However, the appropriate comparator for the vast majority of patients in SA and used in the original article is not 1 year of adjuvant trastuzumab but no treatment with trastuzumab at all.

3. The author makes a series of statements that cost cannot be used as the single basis for decision-making. This is discussed in the original article but is ignored in the letter. Nevertheless, drug costs merit specific consideration because they have a dominant role in determining access for patients.

The author makes a series of aspirational statements about determining cost-effectiveness. These are theoretically correct but do not take account of locally available resources to do these determinations. Moreover, the letter provides no data. Simpler and pragmatic methods, which at least provide data, are helpful in discussions of resource allocation in this difficult area.

The original article sought to be constructive and urges ongoing engagement of the state, healthcare institutions and the pharmaceutical industry to address high drug costs and to progressively bring the benefits from advances in cancer treatment to all patients.

\section{Raymond P Abratt}

Head of Clinical Governance, Independent Clinical Oncology Network Emeritus Professor of Radiation Oncology, University of Cape Town, South Africa raymond.abratt@cancernet.co.za

\footnotetext{
1. Abratt RP. Cost considerations in determining the affordability of adjuvant trastuzumab in breast cancer. S Afr Med J 2016;106(10):981-982. http://dx.doi.org/10.7196/SAMI.2016.v106i10.11141
} 